\title{
THE INTRACARDIAC ELECTROGRAM AS AN AID IN THE LOCALIZATION OF PULMONARY STENOSIS
}

\author{
BY \\ D. EMSLIE-SMITH, K. G. LOWE, AND I. G. W. HILL \\ From the Department of Medicine, University of St. Andrews
}

Received April 29, 1955

The intracardiac electrogram (IEG), as recorded from an electrode at the tip of a cardiac catheter, shows a striking contrast in pattern between that from the lumen of the pulmonary artery and that from the cavity of the right ventricle. The change is well seen in continuous records made during withdrawal of the catheter from the pulmonary artery through the pulmonary valve and is, in general, abrupt. Having regard to the importance of accurate pre-operative diagnosis of the site of the stenosis, and recognizing the artefacts that may complicate pressure tracings, it appeared to us that recording the IEG along with the pressure pulse during catheterization of patients with pulmonary stenosis might be useful in determining the site of the obstruction in the right ventricular outflow tract.

\section{MATERIAL AND Method}

The IEG obtained from a catheter carrying a nickel-silver electrode on its tip was recorded optically on photosensitive paper together with limb- and chest-lead electrocardiograms, and pressure pulses (Emslie-Smith, 1955), the pressures being measured by a capacitance manometer. It was recorded during diagnostic catheterization of 43 patients. Of these, 19 were cases of pulmonary stenosis, simple or complicated. The remaining 24 included 18 with rheumatic heart disease, 1 with constrictive pericarditis, and 5 with congenital heart disease without pulmonary stenosis.

In most of the 19 patients with pulmonary stenosis, several recordings of pressure pulse and $I E G$ were made during repeated withdrawal of the catheter from the pulmonary artery to the right ventricle, to confirm that the change in pattern of the $I E G$ at the pulmonary valve was reproducible in successive tests.

\section{RESULTS}

General. In 4 of the 43 patients, the electrocardiogram showed ventricular arrhythmia as the catheter tip passed through the pulmonary valve, so that the characteristic change in the $I E G$ was obscured. In 37 of the other 39 the change in form of the $I E G$ at the pulmonary valve was abrupt. In two patients the change in the form at this level was much less abrupt; both had right bundlebranch block. There were, however, several cases of right bundle-branch block among the 37 showing an abrupt transition.

Pulmonary Valvular Stenosis (abrupt change in IEG synchronous with rise in systolic pressure at pulmonary valve). In 15 patients the clinical diagnosis was pulmonary valvular stenosis with intact ventricular septum; several of them had proven or suspected atrial septal defects. All had some symptoms referable to the heart disease, although they were handicapped to varying degrees. The resting right ventricular systolic pressures ranged from 23 to $170 \mathrm{~mm}$. Hg. Seven had marked post-stenotic dilatation of the pulmonary artery radiologically. The only patient who was cyanosed at rest had severe pulmonary stenosis probably with an atrial septal defect, and improved considerably following pulmonary valvotomy. No patient had digital clubbing. 
Fig. 1 and 2 show the typical pattern of pressure pulse and $I E G$ in pulmonary valvular stenosis. A marked and, in some cases, dramatic rise in systolic pressure is recorded simultaneously with an abrupt change in the form of the $I E G$.

Recognition of the Venturi Effect in Pulmonary Valvular Stenosis. By contrast with Fig. 1 and 2, Fig. 3, also from a patient with pulmonary valvular stenosis, shows marked pressure oscillations during the withdrawal of the catheter and before the right ventricular pressure is recorded, suggesting, at first sight, the pressure tracing of infundibular stenosis. These pressure pulses, however,

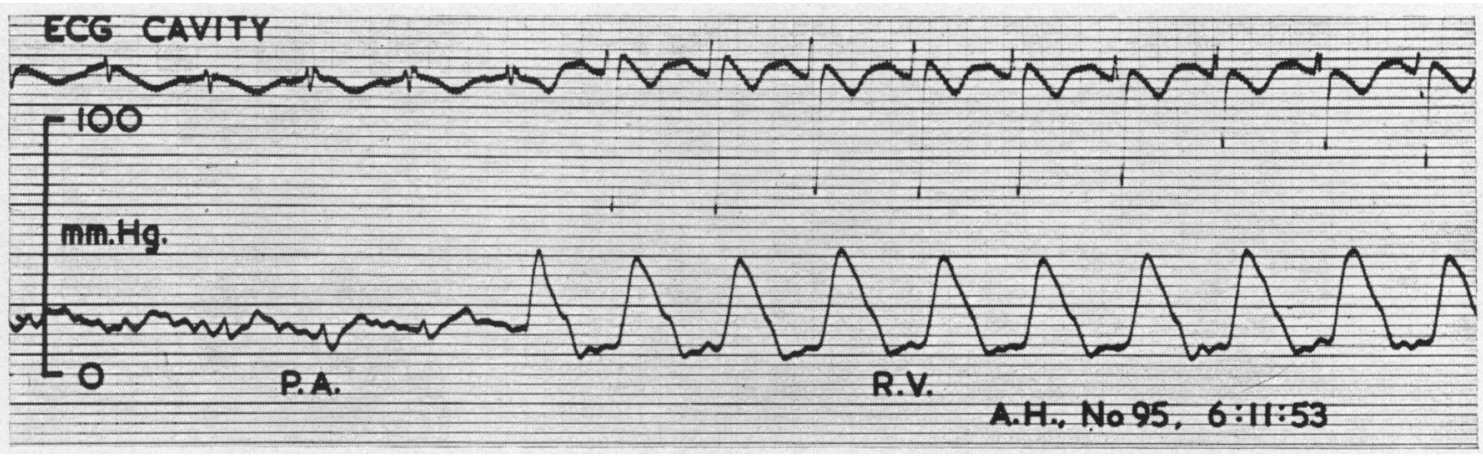

FIG. 1.-Pulmonary valvular stenosis with intact ventricular septum. The catheter tip has passed back through the pulmonary valve during ventricular systole and the first right ventricular pressure pulse is incomplete; the corresponding complex of the $I E G$ shows only minor $T$ wave change as compared with the complexes recorded from the lumen of the pulmonary artery. The subsequent full right ventricular pressure pulses are accompanied by $I E G$ of right ventricular type. A.H., aged 6 years.

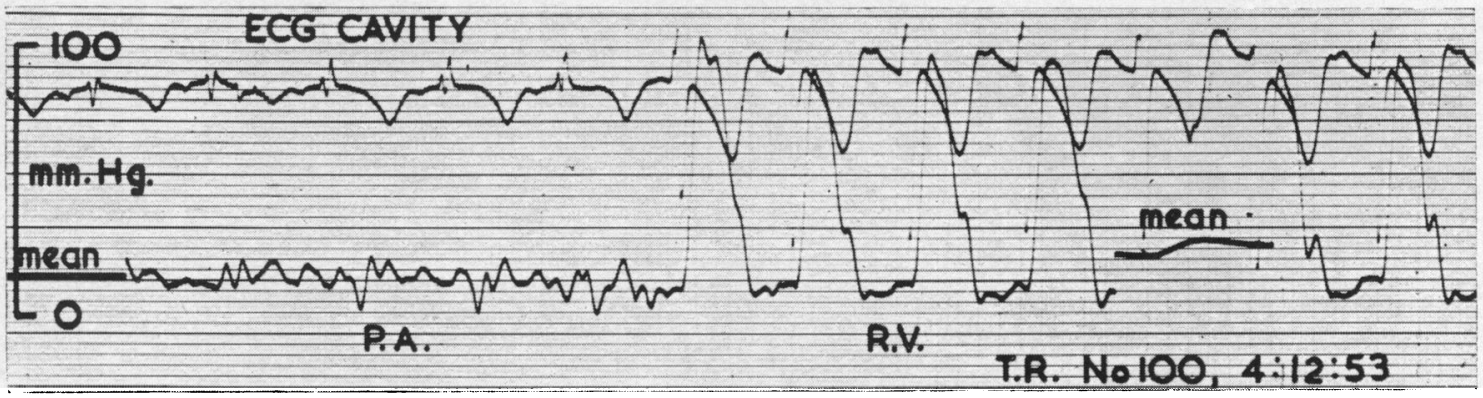

FIG. 2.-Pulmonary valvular stenosis with intact ventricular septum. The catheter tip has passed through the pulmonary valve during ventricular diastole and, synchronous with the recording of the first full right ventricular pulse there is an abrupt change in the IEG to right ventricular type. T.R., aged 9 years.

are recorded from the main pulmonary artery and show a fall instead of a rise during systole, as is obvious from the synchronous $I E G$ tracing. They are, in fact, the "negative pressure waves" that have been ascribed by Sobin et al. (1954) to the Venturi effect produced by the high velocity jet of blood forced through the stenosed pulmonary valve. It will be noted that in the IEG tracing, the first complex of right ventricular pattern, with deeply inverted $T$ wave, was recorded synchronously with the first full right ventricular systolic pressure wave, confirming the valve as the site of the stenosis. This patient showed characteristic post-stenotic dilatation of the pulmonary artery. In another patient with severe pulmonary stenosis, we recorded negative pressure waves of an amplitude of $40 \mathrm{~mm}$. $\mathrm{Hg}$ in the main pulmonary artery (Fig. 4). Again the IEG confirmed that the stenosis was valvular.

Recognition of Positive Pressure Artefact in Main Pulmonary Artery, in Pulmonary Valvular Stenosis. In three patients the diagnosis was Fallot's tetralogy. Records from one of these patients 


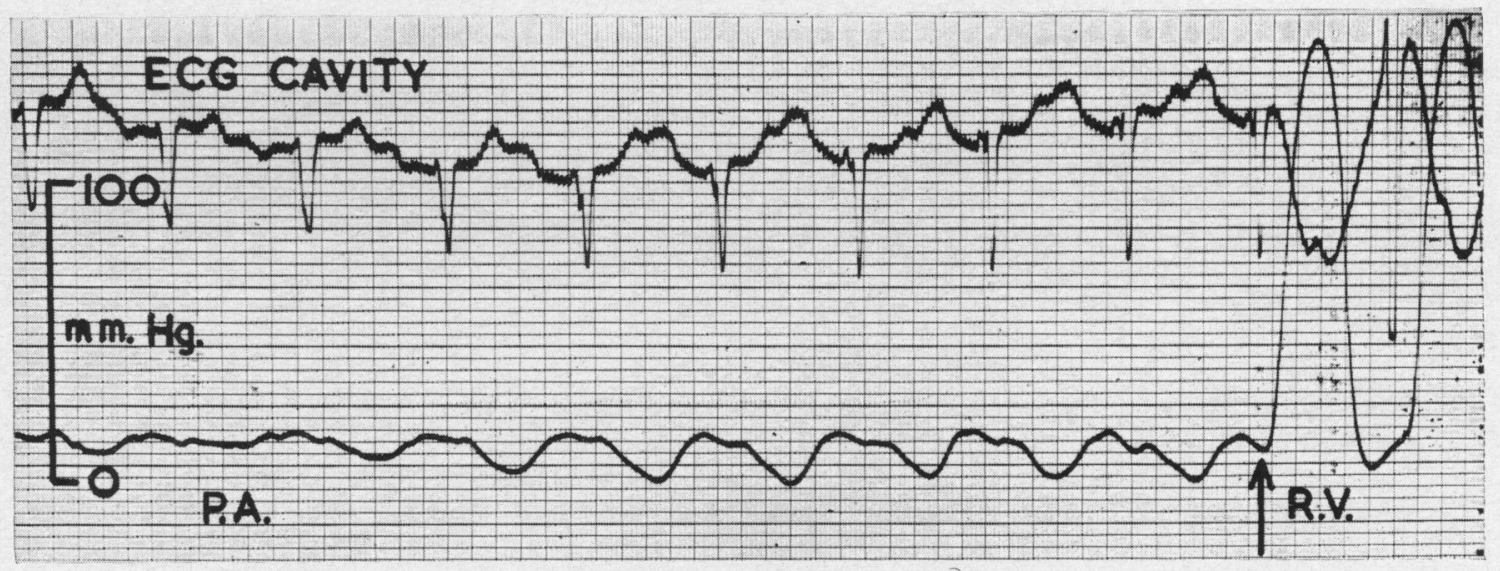

FIG. 3.-Pulmonary valvular stenosis with intact ventricular septum and marked post-stenotic dilatation of main pulmonary artery. As the catheter tip is withdrawn down the main pulmonary artery towards the pulmonary valve there appears a systolic pressure dip-this should not be confused with the diastolic pressure dip recorded when the catheter tip passes through a normal pulmonary valve. The passage of the catheter tip through the pulmonary valve is shown by the abrupt change in form of the $I E G$ which is synchronous with a large right ventricular pressure pulse. I.S., aged 14 years.

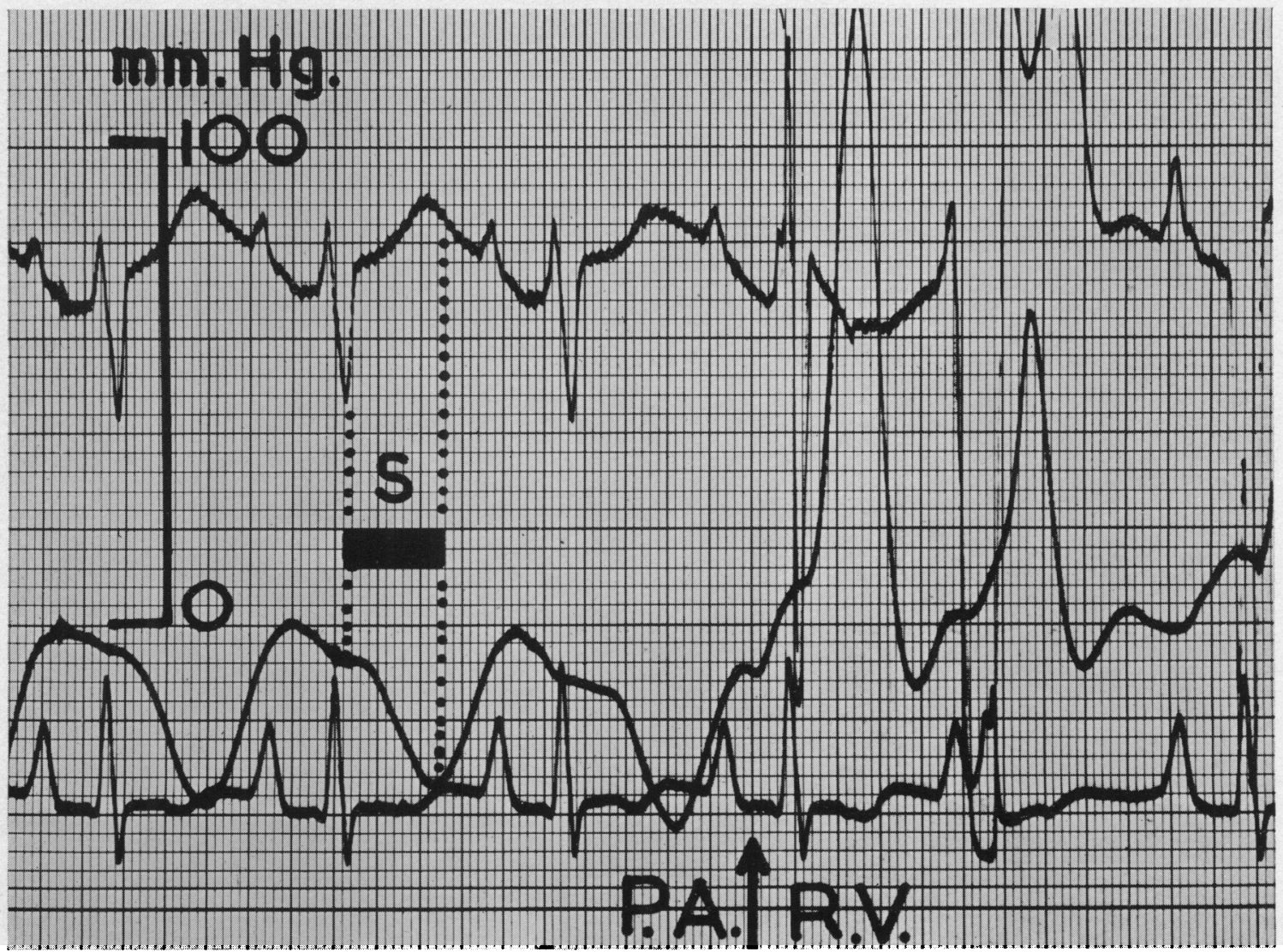

FIG. 4.-Pulmonary valvular stenosis with intact ventricular septum. The tracings from above downwards are: $I E G$, pressure pulse, and standard electrocardiogram lead II. S=systole. Note the systolic pressure dip in the main pulmonary artery and the change in $I E G$ as the first right ventricular pulse is recorded. J.M., aged 11 years. 


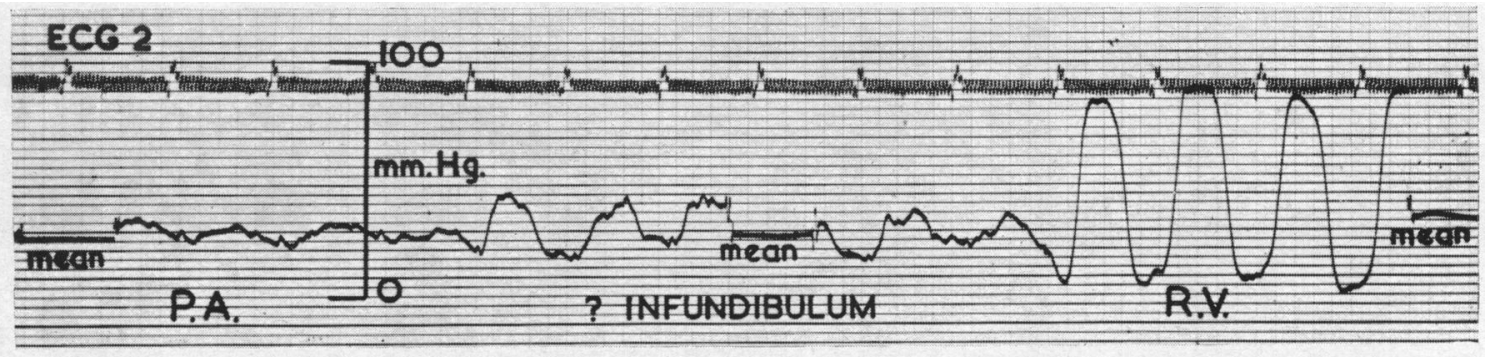

FIG. 5.-Fallot's tetralogy. Tracing obtained at cardiac catheterization in 1953 . The provisional diagnosis was combined valvular and infundibular pulmonary stenosis. H.C., aged 5 years.

illustrate another possible fallacy in the localization of pulmonary stenosis from pressure tracings. When the first pressure tracing was recorded without an $I E G$, its form suggested combined valvular and infundibular stenosis (Fig. 5). Later, the manœuvre was repeated with an electrode-tipped catheter, permitting synchronous recording of pressure pulses and IEG (Fig. 6). It was noted that

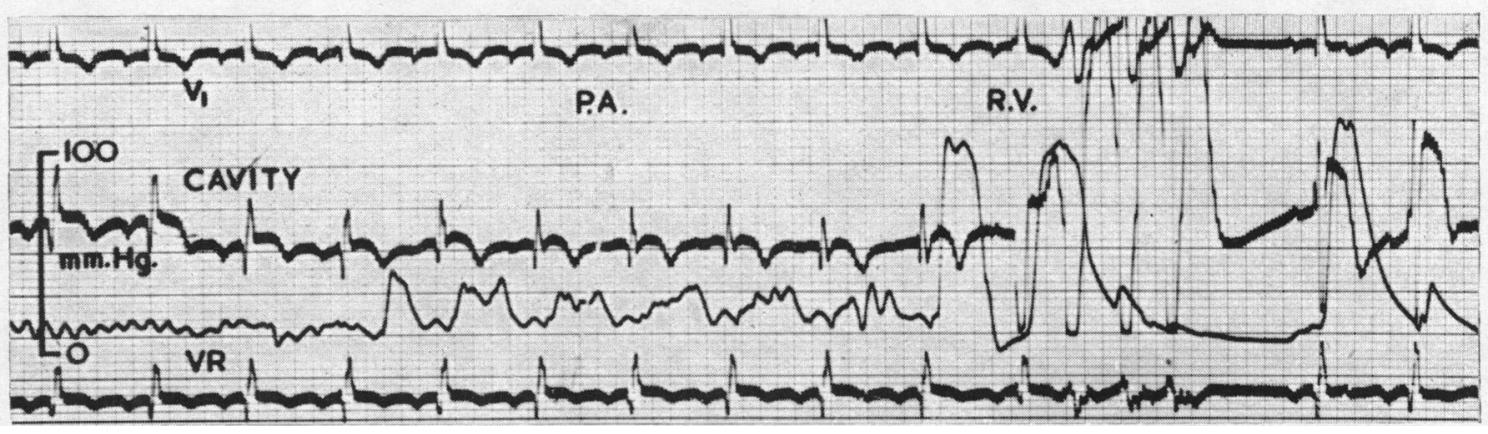

FIG. 6.-Cardiac catheterization repeated in 1955 20/1/55. Tracings from above downwards: electrocardiogram (V1), IEG, pressure pulse, and lead VR. Reading from left to right there is an increase in the amplitude of pressure pulse after the fourth QRS complex, without any accompanying change in the IEG. There is a minor change in the $T$ wave of the tenth IEG complex synchronous with the first right ventricular pressure pulse. There follow four ventricular ectopic beats. The succeeding sinus beat shows the right ventricular type of $I E G$ complex. Pulmonary valvular stenosis (as part of a Fallot's tetralogy) was therefore diagnosed and this was confirmed at pulmonary valvotomy.

an abrupt change in the IEG did not occur before the appearance of the larger systolic pressure waves of nearly $100 \mathrm{~mm}$. $\mathrm{Hg}$ amplitude. The much smaller rise in systolic pressure, seen in the left-hand side of Fig. 6, therefore occurred distal to the pulmonary valve. Valvular pulmonary stenosis was diagnosed as the only obstruction to the right ventricular outflow tract, and this was confirmed at operation when valvotomy was successfully performed. At the second catheterization it had been noted that, with simultaneous screening of the heart and monitoring of pressure pulse and IEG on the oscilloscope, the larger pulmonary systolic pressure waves were seen when the tip of the catheter lay in the main pulmonary artery and the amplitude diminished markedly when the catheter tip entered the right or left main branch. The increased amplitude of the pressure pulse in the main pulmonary artery may have been caused by pressure artefact due to vigorous movement of the catheter along the axis of its tip (Wood et al., 1954).

The IEG in Infundibular Stenosis. The last patient with pulmonary stenosis was an asymptomatic 16-year-old girl with a widespread harsh systolic murmur and thrill, maximal at the left sternal border in the third and fourth interspaces. Cardiac catheterization showed that she had a ventricular septal defect with left-to-right shunt. In addition, a systolic pressure gradient was demonstrated, during the passage of the catheter through the body of the right ventricle (Fig. 7). The manœuvre 


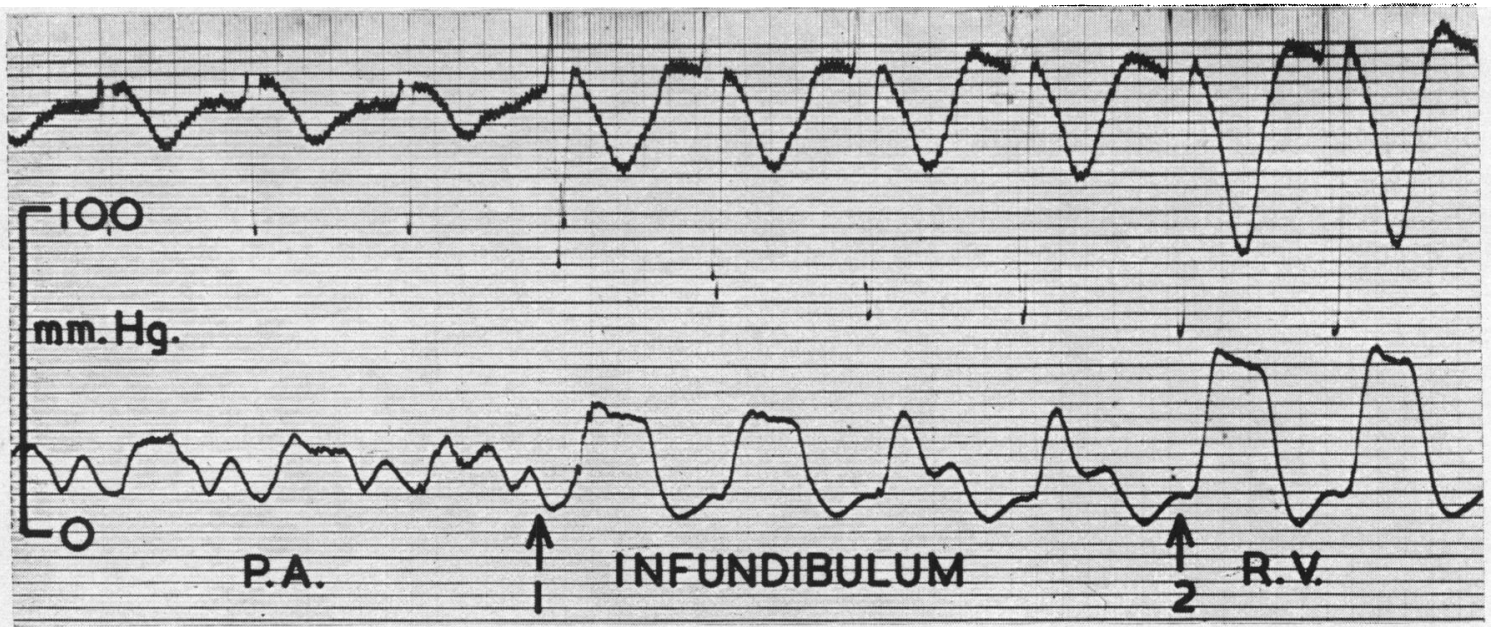

FIG. 7.-Pulmonary infundibular stenosis with ventricular septal defect (left-to-right shunt). Recording of pressure pulses and IEG at cardiac catheterization. At the first arrow there is a small systolic pressure gradient synchronous with a change in the $I E G$ to right ventricular type. At the second arrow, there is a further systolic pressure gradient with little further change in IEG. Similar tracings were obtained on three separate withdrawals of the catheter from pulmonary artery to right ventricle. The tracings suggest infundibular stenosis. C.T., aged 16 years.

was repeated several times and this systolic pressure gradient was a constant finding. From simultaneous screening and monitoring the pressure pulse and $I E G$, it was thought that there was an infundibular stenosis about one inch below the pulmonary valve. Anatomical proof of this case, however, is lacking.

\section{Discussion}

The interpretation of pressure pulse tracings recorded during cardiac catheterization of patients with pulmonary stenosis is often difficult. In the first place, the systolic pressure change should be abrupt and of impressive size (Shephard, 1954). To be significant, positive pressure pulses should be systolic in timing. Thus, careful inspection will detect those pressure pulses that are negative during systole and are caused by the tip of the catheter lying in the high velocity jet of blood flowing through the pulmonary valve (Sobin et al., 1954). Conversely, not all positive systolic pressure pulses are free of artefact for Wood et al. (1954) have demonstrated positive pressure increments in the pulmonary arterial pressure pulse due to forward movement of the catheter tip in systole. We have found the $I E G$ to be of help in the interpretation of the pressure pulse tracings since, as we have shown, the abrupt change in form of the IEG recorded from the catheter electrode is generally a signal of its passage through the valve. If such an abrupt change in form is synchronous with a significant rise in systolic pressure during withdrawal of an electrodetipped catheter, valvular stenosis can be diagnosed with reasonable confidence.

On the other hand, during withdrawal of a catheter in a patient with infundibular stenosis, one may expect the abrupt change in form of the $I E G$, signalling the passage of the tip through the valve, to precede the rise in systolic pressure occurring at the junction of infundibulum and main right ventricular cavity.

\section{SUMMARY}

When an electrode-tipped cardiac catheter is withdrawn from the pulmonary artery back into the right ventricle, an abrupt change in form of the intracardiac electrogram usually occurs at the region of the pulmonary valve. Simultaneous recording of the intracardiac electrogram and 
pressure pulse in patients with pulmonary stenosis is thus of help in locating the level of the stenosis. In valvular stenosis the systolic pressure gradient is related to the abrupt change in form of the electrogram. Artefacts in the pressure tracing that can lead to a wrong diagnosis of valvular stenosis as infundibular stenosis are negative pressure waves due to Venturi effect and, possibly, systolic pressure increments due to catheter movement, when the catheter tip lies just beyond the pulmonary valve. Such errors in diagnosis may be obviated by correlation of pressure pulse and intracardiac electrogram. In infundibular stenosis, during withdrawal of the catheter the abrupt change in form of the intracardiac electrogram, signalling passage through pulmonary valve, should precede the rise in systolic pressure occurring at the junction of infundibulum and main right ventricular cavity.

We are grateful to Dr. C. Pickard, Consultant Radiologist, Dundee Royal Infirmary, and members of his staff for their interest and co-operation in this work; to Professor D. M. Douglas for the operative findings in the two cases quoted; and to the staff of the Department of Medicine for secretarial and photographic assistance.

\section{REFERENCES}

Emslie-Smith, D. (1955). Brit. Heart J., 17, 219.

Shephard, R. J. (1954). Brit. Heart J., 16, 361.

Sobin, S. S., Carson, M. J., Johnson, J. L., and Baker, C. R. (1954). Amer. Heart J., 48, 416.

Wood, E. H., Leusen, I. R., Warner, H. R., and Wright, J. L. (1954). Circulation Research, 2, 294. 